\title{
Modelo numérico do transporte de água e soluto no solo: I - simulação da distribuição de umidade
}

\author{
Marcus M. Corrêa ${ }^{1}$, Mauro A. Martinez ${ }^{2}$, Luiz C. Costa ${ }^{2}$, Hugo A. Ruiz ${ }^{2}$, Marcelo M. Corrêa ${ }^{3}$ \& Silvio C. Sampaio ${ }^{4}$
}

\begin{abstract}
RESUMO
As equações diferenciais do movimento de água e do transporte de soluto em solo não saturado, considerando-se a existência de extração pela planta, foram resolvidas utilizando a técnica diferenças finitas. Para a implementação do modelo desenvolveu-se um programa em linguagem Delphi, denominado SIMASS-C - SImulação do Movimento de Água e Soluto no Solo, considerando-se a presença de Cultura. O modelo fornece, em diferentes tempos, os valores de umidade, do potencial matricial, do fluxo da água e da concentração de soluto ao longo do perfil do solo. Obtém-se, ainda, como resultados de saída, o crescimento e a densidade das raízes, o índice de área foliar e a evapotranspiração da cultura. Para testar o modelo desenvolvido conduziu-se um experimento em casa de vegetação, onde 42 colunas de solo foram montadas em tubo de PVC rígido. Em cada coluna, a cultura do milho foi semeada e durante 30 dias após a germinação, a umidade do solo e o desenvolvimento da cultura foram monitorados. Os resultados experimentais mostraram, ao nível de probabilidade de 90\%, que o modelo SIMASS-C foi preciso em simular o transporte de água no solo.
\end{abstract}

Palavras-chave: diferenças finitas, método iterativo, SIMASS-C

\section{Numerical model for water and solute transport in the soil: I - simulation of the moisture distribution}

\begin{abstract}
The differential equations that govern the water flow and the solute transport in an unsaturated soil, considering the water extraction by plants were solved using the finite difference method. A computer model named SIMASS-C (Simulation of the water and solute transport in the soil considering water extraction) was developed using Delphi language. The model allows to calculate the water content, matric potential, water flux and solute concentration through the soil profile. Besides that, the model output gives the growth and the density of the roots, the leaf area index and the crop evapotranspiration. To test the model, an experiment was conducted in a green house using 42 soil columns made of PVC tubes. In each column, corn was seeded and during 30 days after the emergence the soil water content was determined. The SIMASS-C was used to simulate the soil water content during the experiment. By comparing the simulated and the experimental data, it was possible to conclude that the SIMASS-C did quite well in predicting the soil water movement in the soil.
\end{abstract}

Key word: finite differences, iterative method, SIMASS-C

1 UFRPE, Rua Dom Manoel de Medeiros s/n, CEP: 52171-030, Recife, PE, Fone: (081)3302-1261, metri@ufrpe.br 2 UFV, Av. P.H. Rolfs s/n, Viçosa, MG, CEP: 36570-000, (031)3899-1910, mmauro@ufv.br, l.costa@ufv.br hruiz@ufv.br, 3 UNEC, Av. Moacir de Mattos, n. 49, CEP: 35300-047, Caratinga, MG, Fone: (031)3871-5450 marcelometri@yahoo.com ${ }^{4}$ UNIOESTE, Rua Universitária n. 1619, Cascavel, PR, CEP: 85819-110, Fone: (045)220-3000, ssampaio@unioeste.br 


\section{INTRODUÇÃO}

O crescimento da produção agrícola exige transformações com inovações tecnológicas que permitam a melhoria da produtividade das culturas. Um dos desafios mais significativos da agricultura atual é o aumento da competitividade associada à preservação do meio ambiente, permitindo benefícios sustentáveis nas explorações agrícolas. Neste contexto, é conveniente se avaliar e adequar cada um dos fatores que compõem o sistema de produção, inclusive o manejo da água de irrigação. Os modelos matemáticos surgem como ferramenta útil, possíveis de serem aplicados na definição de quanto e quando se deve proceder a irrigação, racionalizando a operação e tornando-a mais eficiente em seus vários aspectos. Wagenet \& Hutson (1994) e Sagardoy (1993) consideram que os avanços computacionais aliados aos métodos numéricos têm influenciado positivamente a modelagem, permitindo melhor entendimento dos processos naturais que governam o transporte de água no solo.

Como exemplos de modelos matemáticos avançados em que se utilizam técnicas numéricas, cita-se o modelo WAVE (Water and Agrochemicals in soil, crop and Vadose Environment), desenvolvido por Vanclooster et al. (1994) e o HYDRUS, desenvolvido por Vogel et al. (1996). Nesses modelos além de se considerar o transporte dinâmico de substâncias químicas no solo, inclusive os processos de transformação, leva-se em conta, ainda, a presença de um sistema radicular ativo, o que promove, conseqüentemente, resultados de simulação mais realísticos.

Recentemente, o Brasil começou a atuar nesse campo de pesquisa e, nos trabalhos de Costa (1998) e Oliveira et al. (2000) buscou-se desenvolver modelos capazes de predizer o transporte de água e solutos no solo; verifica-se, no entanto, que os modelos desenvolvidos pelos autores não contemplaram, pelo menos de modo satisfatório, o transporte de água e solutos em condições não-saturadas. Oliveira \& Lima (2003) desenvolveram um modelo numérico para simular o transporte de água em solo não-saturado, mas consideraram o solo sem cultura e hidraulicamente homogêneo.

Ante o exposto objetivou-se, com o presente trabalho:

- Desenvolver um modelo matemático computacional capaz de calcular a distribuição da umidade e da concentração de solutos no perfil do solo hidraulicamente heterogêneo, sob condições de escoamento não-saturado, levando-se em consideração a presença de sistema radicular ativo.

- Testar o modelo desenvolvido comparando-se os resultados obtidos por simulação com os encontrados em ensaios experimentais, em colunas de solo.

\section{MATERIAL E MÉTODOS}

\section{Descrição do Modelo}

O modelo SIMASS-C (프mulação do Movimento de Água

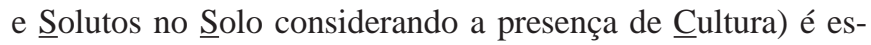
sencialmente unidimensional vertical, visto que considera a existência de heterogeneidade do solo, na forma de camadas,

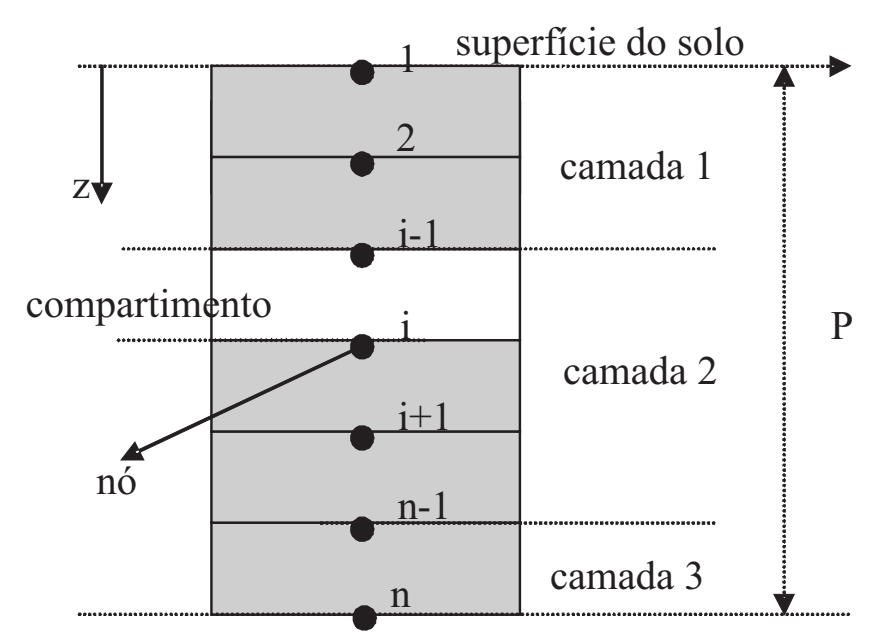

Figura 1. Esquema da região física utilizada para simulação pelo modelo SIMASS-C, discretizada em " $n$ " nós

divididas em espaços denominados compartimentos. No contorno de cada compartimento são especificados dois nós para que sejam utilizados pelas técnicas de diferenças finitas. A Figura 1 apresenta uma visão esquemática da região física estudada, inclusive o sistema de orientação empregado.

O movimento de água no perfil do solo é analisado através da equação de Richards, considerando-se o escoamento unidimensional não-saturado e a coordenada vertical orientada positivamente para baixo:

$$
\frac{\partial \theta}{\partial \mathrm{t}}=\frac{\partial}{\partial \mathrm{z}}\left[\mathrm{K}(\theta)\left(\frac{\partial \mathrm{h}}{\partial \mathrm{z}}-1\right)\right]+\mathrm{R}
$$

em que " $\theta$ ” é a umidade volumétrica do solo $\left(\mathrm{L}^{3} \mathrm{~L}^{-3}\right)$, “h” é o potencial matricial (L), "K” é a condutividade hidráulica do solo $\left(\mathrm{L} \mathrm{T}^{-1}\right)$ e "R" é o termo fonte/sumidouro utilizado na simulação da extração de água pelas culturas $\left(\mathrm{T}^{-1}\right)$. Introduzindo-se o termo capacidade hídrica específica, tem-se:

$$
\mathrm{E}(\mathrm{h}) \frac{\partial \mathrm{h}}{\partial \mathrm{t}}=\frac{\partial}{\partial \mathrm{z}}\left[\mathrm{K}(\mathrm{h})\left(\frac{\partial \mathrm{h}}{\partial \mathrm{z}}-1\right)\right]+\mathrm{R}
$$

A equação de Richards foi resolvida de forma iterativa, em duas etapas, usando-se diferenças finitas. Inicialmente, uma primeira estimativa do potencial matricial é encontrada utilizando-se um esquema de discretização implícita com linearização explícita da Eq. 2; em seguida, é realizado um refinamento dessa solução pelo processo iterativo de NewtonRaphson, com um esquema de discretização implícita da Eq. 1. Em ambos os casos, as soluções das equações fornecem um sistema tridiagonal de equações lineares que é resolvido pelo algoritmo de Thomas. A solução numérica obtida no refinamento é apresentada a seguir:

$$
a_{i}\left(h_{i-1}^{j+1}\right)^{\tau+1}+b_{i}\left(h_{i}^{j+1}\right)^{\tau+1}+c_{i}\left(h_{i+1}^{j+1}\right)^{\tau+1}=d_{i}
$$

em que: 


$$
\begin{aligned}
& \mathrm{a}_{\mathrm{i}}=-\frac{\Delta \mathrm{t}}{\Delta \mathrm{z}^{2}}\left(\mathrm{~K}_{\mathrm{i}-1 / 2}^{\mathrm{j}+1}\right)^{\tau} \\
& \mathrm{b}_{\mathrm{i}}=\left(\mathrm{E}_{\mathrm{i}}^{\mathrm{j}+1}\right)^{\tau}+\frac{\Delta \mathrm{t}}{\Delta \mathrm{z}^{2}}\left(\mathrm{~K}_{\mathrm{i}+1 / 2}^{\mathrm{j}+1}\right)^{\tau}+\frac{\Delta \mathrm{t}}{\Delta \mathrm{z}^{2}}\left(\mathrm{~K}_{\mathrm{i}-1 / 2}^{\mathrm{j}+1}\right)^{\tau} \\
& \mathrm{c}_{\mathrm{i}}=-\frac{\Delta \mathrm{t}}{\Delta \mathrm{z}^{2}}\left(\mathrm{~K}_{\mathrm{i}+1 / 2}^{\mathrm{j}+1}\right)^{\tau} \\
& \mathrm{d}_{\mathrm{i}}=\left(\mathrm{E}_{\mathrm{i}}^{\mathrm{j}+1} \mathrm{~h}_{\mathrm{i}}^{\mathrm{j}+1}\right)^{\tau}-\left(\left(\theta_{\mathrm{i}}^{\mathrm{j}+1}\right)^{\tau}-\theta_{\mathrm{i}}^{\mathrm{j}}\right)-\frac{\Delta \mathrm{t}}{\Delta \mathrm{z}}\left(\mathrm{K}_{\mathrm{i}+1 / 2}^{\mathrm{j}+1}\right)^{\tau}+\frac{\Delta \mathrm{t}}{\Delta \mathrm{z}}\left(\mathrm{K}_{\mathrm{i}-1 / 2}^{\mathrm{j}+1}\right)^{\tau}+\mathrm{R}_{\mathrm{i}}^{\mathrm{j}} \Delta \mathrm{t}
\end{aligned}
$$

em que "i” representa a posição do nó na malha de discretização, “j” o incremento do tempo, e o sobrescrito " $\tau$ ” refere-se à ordem da iteração.

O processo iterativo é resolvido até que a convergência desejada seja alcançada. No modelo SIMASS-C, a interface com o usuário permite a escolha de dois critérios de convergência. É possível utilizar, como controle iterativo, o erro absoluto (EA) ou erro relativo (ER).

$$
\begin{gathered}
\operatorname{abs}\left(\left(h_{i}^{j+1}\right)^{\tau}-\left(h_{i}^{j+1}\right)^{\tau+1}\right) \leq E A \\
\operatorname{abs}\left(\frac{\left(\left(h_{i}^{j+1}\right)^{\tau}-\left(h_{i}^{j+1}\right)^{\tau+1}\right)}{\left(h_{i}^{j+1}\right)^{\tau}}\right) \leq E R
\end{gathered}
$$

O sistema de equações (3) é aplicado apenas aos nós centrais, $2 \leq \mathrm{i}<\mathrm{n}-1$. Para os nós extremos deve-se incorporar as condições de contorno do problema.

\section{a) Condição de contorno tipo Dirichlet}

Neste caso, os valores de potencial matricial do primeiro e do último nó, são conhecidos em qualquer tempo. Conseqüentemente, o sistema de equações (3) está delimitado pelos nós $\mathrm{i}=2$ e i $=$ n-1 e as equações para esses nós se tornam, respectivamente:

$$
\mathrm{b}_{2}\left(\mathrm{~h}_{2}^{\mathrm{j}+1}\right)^{\tau+1}+\mathrm{c}_{2}\left(\mathrm{~h}_{3}^{\mathrm{j}+1}\right)^{\tau+1}=\varepsilon_{\mathrm{i}}
$$

em que:

$$
\begin{aligned}
\varepsilon_{2}=d_{2}-a_{2}\left(h_{1}^{j+1}\right)^{\tau+1} & \\
& a_{n-1}\left(h_{n-2}^{j+1}\right)^{\tau+1}+b_{n-1}\left(h_{n-1}^{j+1}\right)^{\tau+1}=\varepsilon_{n-1}
\end{aligned}
$$

em que:

$$
\varepsilon_{\mathrm{n}-1}=d_{\mathrm{n}-1}-\mathrm{C}_{\mathrm{n}-1}\left(\mathrm{~h}_{\mathrm{n}}^{\mathrm{j}+1}\right)^{\tau+1}
$$

\section{b) Condição de contorno tipo Neumann}

Para esta condição, os valores de potencial matricial do primeiro e do último nó, não são conhecidos. O sistema de equações (3), para os nós extremos, torna-se igual a:

$$
\left(\mathrm{a}_{1}+\mathrm{b}_{1}\right)\left(\mathrm{h}_{1}^{\mathrm{j}+1}\right)^{\tau+1}+\mathrm{c}_{1}\left(\mathrm{~h}_{2}^{\mathrm{j}+1}\right)^{\tau+1}=\mathrm{e}_{1}
$$

em que:

$$
\begin{gathered}
\mathrm{a}_{1}+\mathrm{b}_{1}=\left(\mathrm{E}_{1}^{\mathrm{j}+1}\right)^{\tau}+\frac{\Delta \mathrm{t}}{\Delta \mathrm{z}^{2}}\left(\mathrm{~K}_{1+1 / 2}^{\mathrm{j}+1}\right)^{\tau} \\
\mathrm{c}_{1}=-\frac{\Delta \mathrm{t}}{\Delta \mathrm{z}^{2}}\left(\mathrm{~K}_{1+1 / 2}^{\mathrm{j}+1}\right)^{\tau} \\
\mathrm{e}_{1}=\left(\mathrm{E}_{1}^{\mathrm{j}+1} \mathrm{~h}_{1}^{\mathrm{j}+1}\right)^{\tau}-\left(\left(\theta_{1}^{\mathrm{j}+1}\right)^{\tau}-\theta_{1}^{\mathrm{j}}\right)-\frac{\Delta \mathrm{t}}{\Delta \mathrm{z}}\left(\mathrm{K}_{1+1 / 2}^{\mathrm{j}+1}\right)^{\tau}+\frac{\Delta \mathrm{t}}{\Delta \mathrm{z}} \mathrm{q}_{\mathrm{s}}+\Delta \mathrm{t} \mathrm{R}_{1}^{\mathrm{j}} \\
\mathrm{a}_{\mathrm{n}}\left(\mathrm{h}_{\mathrm{n}-1}^{\mathrm{j}+1}\right)^{\tau+1}+\left(\mathrm{b}_{\mathrm{n}}+\mathrm{c}_{\mathrm{n}}\right)\left(\mathrm{h}_{\mathrm{n}}^{\mathrm{j}+1}\right)^{\tau+1}=\mathrm{f}_{\mathrm{n}}
\end{gathered}
$$

em que:

$$
\begin{aligned}
& \mathrm{a}_{\mathrm{n}}=-\frac{\Delta \mathrm{t}}{\Delta \mathrm{z}^{2}}\left(\mathrm{~K}_{\mathrm{n}-1 / 2}^{\mathrm{j}+1}\right)^{\tau} \\
& \mathrm{b}_{\mathrm{n}}+\mathrm{c}_{\mathrm{n}}=\left(\mathrm{E}_{1}^{\mathrm{j}+1}\right)^{\tau}+\frac{\Delta \mathrm{t}}{\Delta \mathrm{z}^{2}}\left(\mathrm{~K}_{\mathrm{n}-1 / 2}^{\mathrm{j}+1}\right)^{\tau} \\
& \mathrm{f}_{\mathrm{n}}=\left(\mathrm{E}_{\mathrm{n}}^{\mathrm{j}+1} \mathrm{~h}_{\mathrm{n}}^{\mathrm{j}+1}\right)^{\tau}-\left(\left(\theta_{\mathrm{n}}^{\mathrm{j}+1}\right)^{\tau}-\theta_{\mathrm{n}}^{\mathrm{j}}\right)+\frac{\Delta \mathrm{t}}{\Delta \mathrm{z}}\left(\mathrm{K}_{\mathrm{n}-1 / 2}^{\mathrm{j}+1}\right)^{\tau}-\frac{\Delta \mathrm{t}}{\Delta \mathrm{z}} \mathrm{q}_{\mathrm{b}}+\Delta \mathrm{tR}_{\mathrm{n}}^{\mathrm{j}}
\end{aligned}
$$

O valor de " $\mathrm{q}_{\mathrm{s}}$ " é dependente das condições de umedecimento e secagem do solo. Para condições de umedecimento, " $\mathrm{q}_{\mathrm{s}}$ " deve ser igual à capacidade de infiltração do solo (CI) se a intensidade de precipitação (IP) for maior que CI, ou igual a IP, caso ocorra o contrário. Durante o período de secagem " "qs" será igual à capacidade de evaporação no solo (CE) se a demanda atmosférica (DA) for maior que CE, ou igual a DA, caso ocorra o contrário.

As capacidades de infiltração e evaporação no solo são calculadas por meio da equação:

$$
\text { CI ou } C E=-\frac{\mathrm{K}_{2}^{\mathrm{j}}+\mathrm{K}_{1}^{\mathrm{j}}}{2}\left[\frac{\mathrm{h}_{2}^{\mathrm{j}}-\mathrm{h}_{1}^{\mathrm{j}}}{\Delta \mathrm{z}}-1\right]
$$

Semelhante ao procedimento adotado por Faria \& Madramootoo (1996), durante o processo de infiltração, para efeito de cálculo de "CI", o valor da condutividade hidráulica do solo para o nó $\mathrm{i}=1$, é considerado igual à condutividade hidráulica do solo saturado.

Relacionando-se, agora, ao último nó no modelo SIMASSC, quando o valor de " $\mathrm{q}_{\mathrm{b}}$ ” não for fixado pelo usuário, o mesmo será calculado considerando-se as condições de escoamento no último compartimento da região estudada, isto é:

$$
\mathrm{q}_{\mathrm{b}}=-\frac{\mathrm{K}_{\mathrm{n}}^{\mathrm{j}}+\mathrm{K}_{\mathrm{n}-1}^{\mathrm{j}}}{2}\left[\frac{\mathrm{h}_{\mathrm{n}}^{\mathrm{j}}-\mathrm{h}_{\mathrm{n}-1}^{\mathrm{j}}}{\Delta \mathrm{z}}-1\right]
$$




\section{Procedimento experimental}

Para testar o modelo desenvolvido, conduziu-se um experimento semelhante ao adotado por Azevedo et al. (1996), em casa de vegetação do Departamento de Solos da Universidade Federal de Viçosa (UFV), durante os meses de novembro e dezembro de 2001.

Em todas as parcelas experimentais utilizaram-se amostras homogêneas de solo, permitindo-se, desta forma, uma avaliação melhor do modelo de transporte desenvolvido. Segundo Lemley et al. (1988) muitos problemas relacionados à quantificação do transporte de contaminantes no campo se devem à impossibilidade de controle das condições de variabilidade. Sassner et al. (1994) fazem comentários idênticos, afirmando que a condução de experimentos em condições reais de campo sofre pela heterogeneidade exibida pelos solos, que ocasiona uma significativa variabilidade do fluxo e dos parâmetros de transporte.

O material de solo empregado foi retirado de um Latossolo Vermelho-Amarelo álico (LVa), localizado nas proximidades da cidade de Viçosa, MG. As amostras do horizonte B foram secadas ao ar e passadas em peneira com malha de $2 \mathrm{~mm}$ (terra fina secada ao ar, TFSA); uma parte da amostra foi separada para determinação da curva de retenção de água do solo, utilizando-se o equipamento de placa de pressão; as demais características do solo utilizadas como dados de entrada do modelo, apresentadas na Tabela 1, foram obtidas no laboratório de Química e Física do Solo da própria Universidade, de acordo com as recomendações da EMBRAPA (1997).

De posse dos resultados da análise química efetuaram-se a fertilização e a calagem do solo, mantendo-o incubado por 30 dias com umidade próxima à capacidade de campo.

Terminada a incubação, 42 colunas de solo foram montadas em tubo de PVC rígido de 0,5 m de comprimento e 0,25 m de diâmetro externo e dispostas em delineamento inteiramente casualizado. Para facilitar a montagem e posterior análise, as colunas foram formadas por cinco anéis concêntricos de 0,10 m de altura, que foram unidos com auxílio de fita adesiva. Os anéis, que formavam a base das colunas de solo, apresentavam sua borda externa totalmente fechada com filme de PVC. Na parede lateral desses anéis, próximo ao filme de PVC, foram introduzidas mangueiras flexíveis, com o intuito de permitir a coleta da água de drenagem.

Tabela 1. Características físico-hídricas das amostras de solo estudado

\begin{tabular}{lc}
\hline \multicolumn{1}{c}{ Características } & Valor \\
Umidade de saturação $\left(\theta_{\mathrm{s}}, \mathrm{L}^{3} \mathrm{~L}^{-3}\right)$ & 0,58 \\
Umidade de capacidade de campo $\left(\theta_{\mathrm{cc}}, \mathrm{L}^{3} \mathrm{~L}^{-3}\right)$ & 0,43 \\
Umidade de ponto de murcha $\left(\theta_{\mathrm{pm}}, \mathrm{L}^{3} \mathrm{~L}^{-3}\right)$ & 0,29 \\
Umidade residual $\left(\theta_{\mathrm{r}} \mathrm{L}^{3} \mathrm{~L}^{-3}\right)$ & 0,15 \\
Massa específica do solo $\left(\rho_{\mathrm{g}}, \mathrm{kg} \mathrm{m}^{-3}\right)$ & 1130 \\
Condutividade hidráulica do solo saturado $\left(\mathrm{K}_{0}, \mathrm{~m} \mathrm{~s}^{-1}\right)$ & $8,3 \times 10^{-6}$ \\
Dispersividade $(\lambda, \mathrm{m})$ & 0,0055 \\
Curva de Retenção (van Genuchten) & \\
Parâmetro " $\alpha$ " & 0,03278 \\
Parâmetro "n" & 1,5158 \\
\hline
\end{tabular}

O preenchimento do solo foi executado de forma a proporcionar uniformidade e homogeneidade em toda a coluna, adicionando-se o solo em camadas de aproximadamente $2 \mathrm{~cm}$ de espessura e exercendo leve pressão com um disco de madeira, momento em que se procurou estabelecer massa específica do solo próximo às condições reais de campo.

Para uniformizar a umidade inicial, as colunas de solo foram saturadas a partir de sua base, com aplicação de água corrente. Garantida a saturação, as extremidades superiores das colunas foram cobertas com filme plástico, objetivando-se evitar perdas de água por evaporação e as colunas de solo foram deixadas em repouso durante 24 horas, para possibilitar a drenagem total do excesso de água. Esse instante caracterizou a condição inicial para a umidade do solo, ou seja, quando se encerrou a drenagem livre em todo o perfil da coluna.

Uma vez padronizada a umidade inicial do solo, quatro sementes de milho (Zea mays L.), variedade BR-201, foram semeadas em cada coluna de solo. Após a emergência, o que ocorreu aproximadamente quatro dias depois do plantio, promoveu-se o desbaste da cultura, deixando-se apenas uma plântula. A opção pelo milho baseou-se por se tratar de uma cultura que apresenta vasta informação disponível e exige quantidades expressivas de fertilizantes nitrogenados, ao longo do seu ciclo vegetativo.

Durante a condução do experimento com o auxílio de um sistema de gotejamento adaptado, realizou-se a aplicação de lâminas de irrigação em intervalos semanais respeitando-se, nessas ocasiões, a demanda hídrica da cultura e a capacidade de retenção do solo. O cálculo da necessidade hídrica da cultura do milho no período foi determinado por meio da evapotranspiração diária, a qual foi estimada pela pesagem de 12 colunas de solo da parcela experimental. Em cada coluna, a lâmina de água evapotranspirada foi considerada a diferença observada no peso da coluna em duas pesagens consecutivas. Realizou-se a aplicação de duas lâminas de fertirrigação, promovidas pela adição de $\mathrm{KNO}_{3}$, na proporção correspondente a 30 e $90 \mathrm{~kg} \mathrm{~N} \mathrm{ha}{ }^{-1}$.

A determinação da umidade do solo em função do tempo e do espaço, foi realizada em intervalos consecutivos de aproximadamente cinco dias, quando três colunas de solo eram desmontadas, e amostras de solo retiradas nas profundidades de $0,0,05,0,10,0,15,0,20,0,30$ e $0,35 \mathrm{~m}$; este processo destrutivo consumiu 30 colunas durante o experimento. Nas amostragens, o sistema radicular do milho era cuidadosamente separado do solo, por peneiramento, e o material de solo acondicionado em recipientes de alumínio para posterior determinação da umidade do solo, utilizando-se o método gravimétrico (EMBRAPA, 1997).

De posse da distribuição temporal e espacial da umidade, o modelo foi testado comparando-se as umidades obtidas experimentalmente com aquelas simuladas pelo programa computacional.

\section{RESULTADOS E DISCUSSÃO}

Os valores da evapotranspiração real da cultura e as lâminas de irrigação aplicadas durante o ensaio experimental, 
estão apresentados na Figura 2. As três lâminas de irrigação, com magnitude de 12,22, 20,37 e 61,11 mm, foram aplicadas, respectivamente, aos 11, 18 e 24 dias após o plantio. O valor apresentado para a terceira irrigação refere-se ao somatório da lâmina de irrigação com precipitação de aproximadamente $50 \mathrm{~mm}$, quando se procurou ocasionar a drenagem das colunas de solo.

É fácil observar que a análise do transporte de água não foi conduzida durante todos os estágios de crescimento da cultura do milho, encerrando-se o experimento aproximadamente 30 dias após o plantio; ressalta-se que esta medida foi necessária para garantir que o crescimento radicular do milho não sofresse nenhum tipo de restrição na coluna de solo.

As Figuras 3 e 4 representam os perfis de umidade do solo simulados pelo modelo SIMASS-C e observados experimentalmente ao longo do período de desenvolvimento da cultura. As simulações foram realizadas considerando-se condições de contorno tipo fluxo, tanto na superfície como na base da coluna. No caso da Figura 4, são apresentados, também, os resultados obtidos quando se considerou a presença de lençol freático na base da coluna (potencial matricial constante e igual a zero). Para avaliar a performance do modelo, nos dados observados foram inseridos os intervalos de confiança, a nível de probabilidade de 90\%, semelhante aos trabalhos de Dou \& Fox (1995), Johnson et al. (1999). Seguindo-se as recomendações de Liwang et al. (1998), a predição do modelo é considerada precisa se os valores simulados estiverem dentro da faixa de variação do intervalo de confiança.

Analisando-se as Figuras 3 e 4, verifica-se que, no geral, os valores simulados se situaram dentro do intervalo de confiança indicando, de acordo com Liwang et al. (1998), que o modelo foi preciso em simular a umidade do solo.

Desvios dos valores experimentais foram verificados 24, 25, 26 e 27 dias após o plantio, correspondentes aos períodos de 12, 24, 48 e 72 horas após a aplicação da lâmina excessiva de água (Figura 4). A explicação para o menor sucesso do modelo está relacionada, sem dúvida, ao mau funcionamento dos drenos localizados na base das colunas, o que ocasionou acúmulo de água nessa região. Os perfis de umidade observados no experimento, no período, são característicos da presença de lençol freático, tendo em vista que a umidade armazenada em todos os perfis, permaneceu aproximadamente constante,

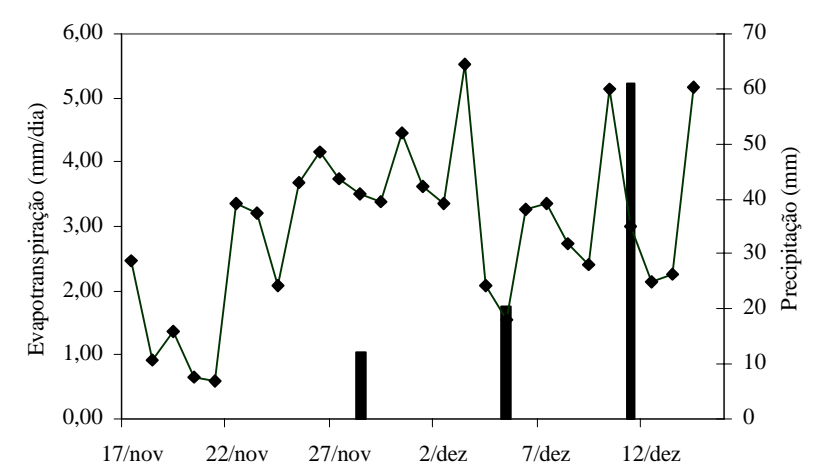

Figura 2. Evapotranspiração real e lâmina de irrigação aplicada ao longo do período de desenvolvimento da cultura
A.

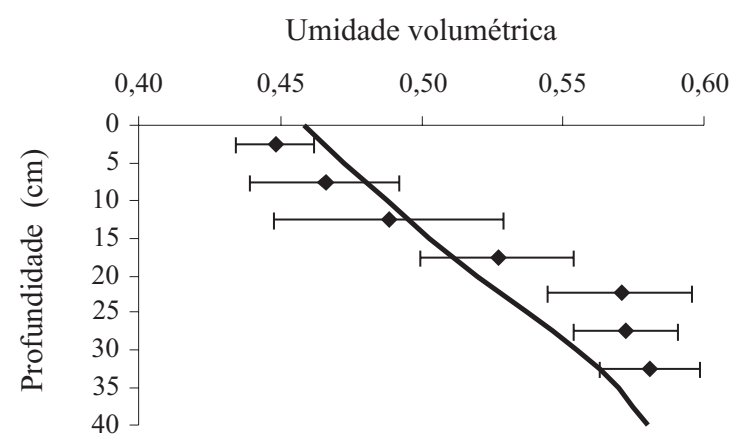

B.

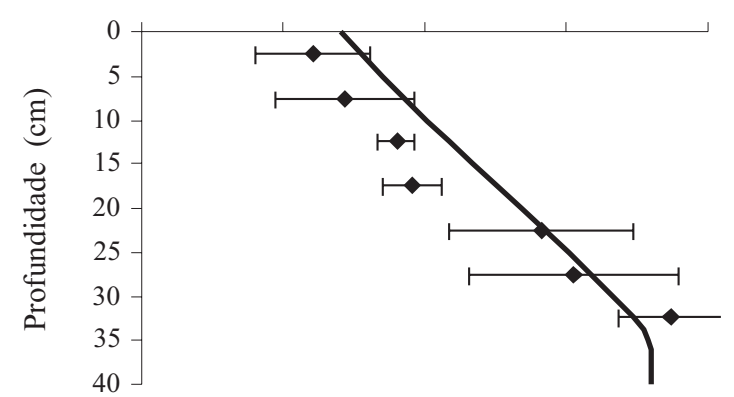

C.

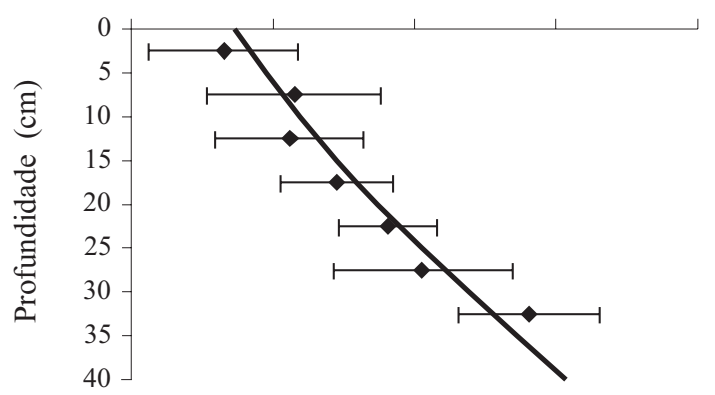

D.

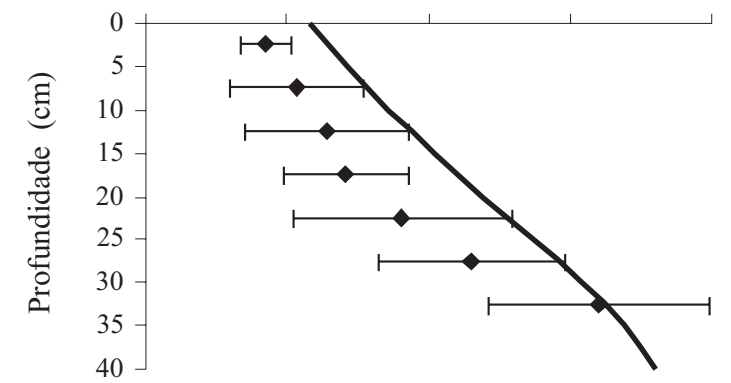

E.

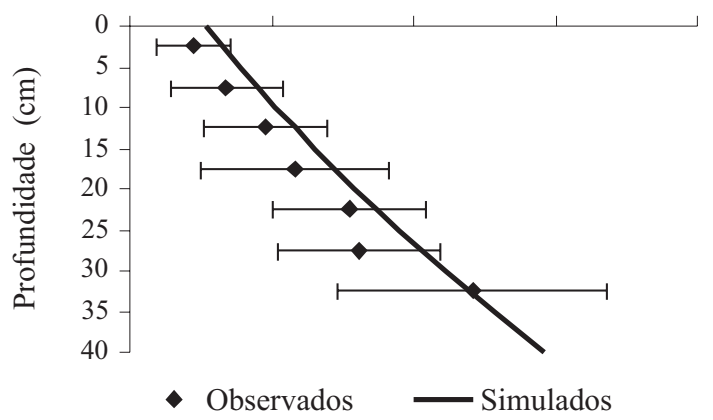

Figura 3. Perfil de umidade do solo observado experimentalmente e simulado pelo Modelo SIMASS-C, respectivamente aos 8 (A), 13 (B), 16 (C), 23 (D) e 24 (E) dias após o plantio, considerando-se fluxo na base da coluna $(\Delta \mathrm{z}=5 \mathrm{~cm}$ e $\Delta \mathrm{t}=0,1 \mathrm{~h})$ 
A. Umidade volumétrica

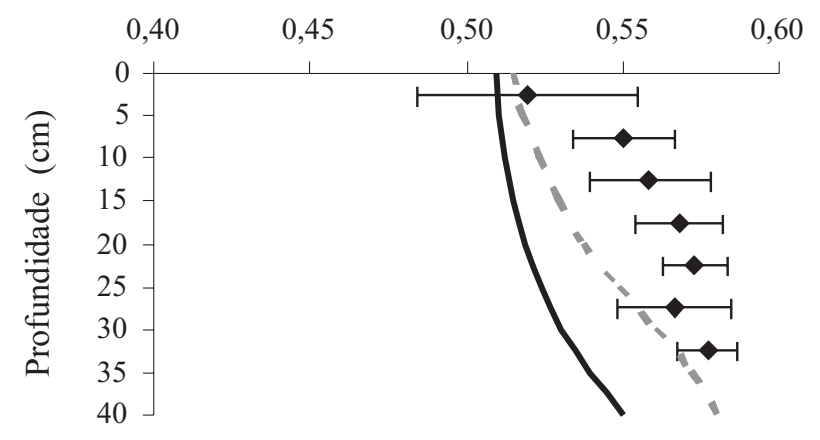

B.

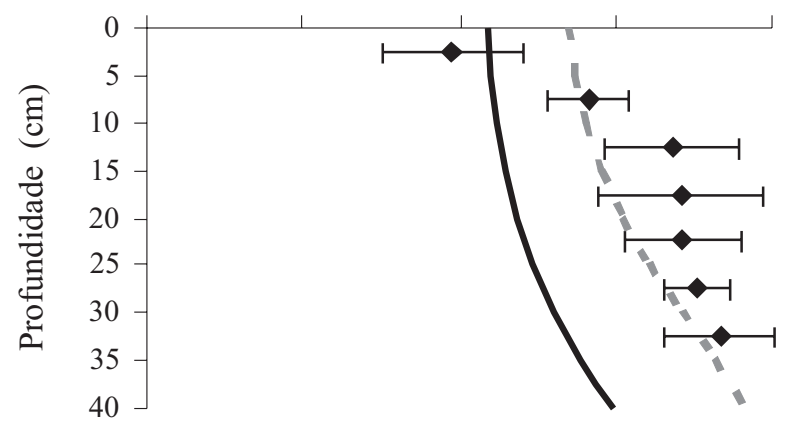

C.

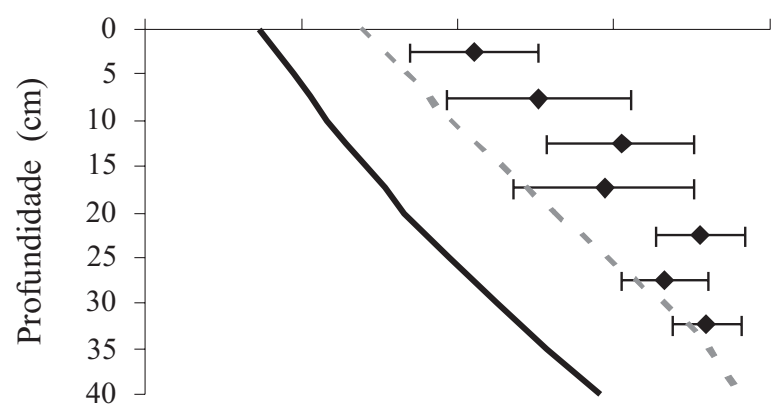

D.

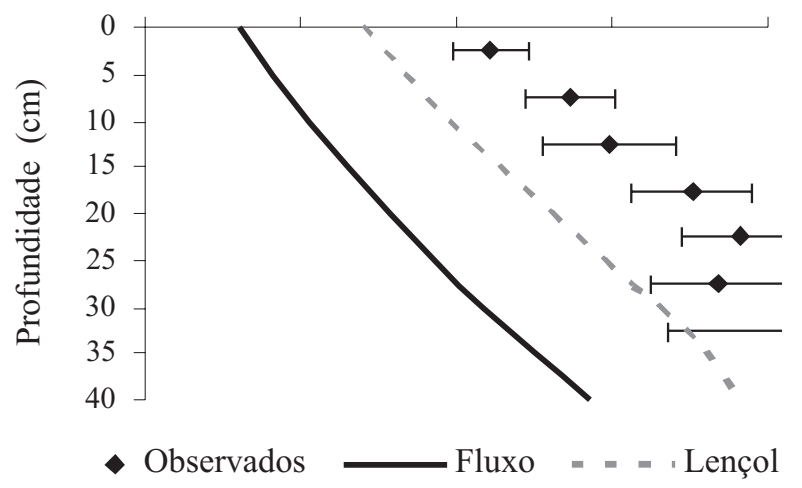

Figura 4. Perfil de umidade do solo observado experimentalmente $\mathrm{e}$ simulado pelo Modelo SIMASS-C, respectivamente 12 (A), 24 (B), 48 (C) e 72(D) h após a aplicação de lâmina excessiva de irrigação, considerandose fluxo na base da coluna $(\Delta \mathrm{z}=5 \mathrm{~cm}$ e $\Delta \mathrm{t}=0,1 \mathrm{~h})$ indicando que a transpiração das plantas e a evaporação no solo foram balanceadas pela ascensão capilar proveniente do acúmulo de água na base da coluna. Considerações semelhantes foram reportadas por Wang et. al. (1998).

Analisando-se, portanto, os perfis simulados e se considerando a presença de lençol freático na base da coluna (Figura 4), verifica-se que a mudança na condição de contorno permitiu uma melhoria sensível nos resultados simulados.

Em uma análise mais minuciosa nas mesmas figuras, pode-se observar que, mesmo considerando a presença de lençol freático, os perfis de umidade estimados pelo modelo apresentaram pontos situados fora do intervalo de confiança das médias observadas. Uma das hipóteses para explicar este comportamento diferenciado poderia estar relacionado a erros associados na medição dos parâmetros de entrada do modelo, provocados pela variabilidade apresentada nas colunas de solo, como justificado por Tillotson \& Wagenet (1982). Tal hipótese se torna mais provável quando se analisam os perfis simulados considerando-se a presença de lençol freático na base da coluna. Na Figura 4 observa-se, no intervalo de profundidade de $30-40 \mathrm{~cm}$, umidade do solo acima da umidade média de saturação utilizada para as simulações no modelo SIMASS-C, que foi de $0,58 \mathrm{~cm}^{3} \mathrm{~cm}^{-3}$. Esta variabilidade, associada a outros prováveis desvios apresentados nos parâmetros de entrada, podem ter influenciado no ajuste dos dados experimentais aos simulados pelo modelo, em condições de umidade do solo próximo da saturação, quando o transporte de água no perfil do solo é maior.

\section{CONCLUSÕES}

1. O modelo SIMASS-C apresentou-se flexível para simular o transporte de água no solo.

2. O modelo SIMASS-C simulou adequadamente, na maioria dos casos, o transporte de água, mostrando-se hábil para predizer a umidade no solo durante o período estudado.

3. Em condições de umidade próxima à saturação, o modelo não apresentou ótimo desempenho.

\section{AGRADECIMENTOS}

Os autores expressam seus maiores agradecimentos ao Conselho Nacional de Desenvolvimento Científico e Tecnológico - CNPq, e à Fundação de Amparo à Pesquisa de Minas Gerais - FAPEMIG.

\section{LITERATURA CITADA}

Azevedo, A. S.; Kanwar, R. S.; Singh, P.; Pereira, L. S. Movement of $\mathrm{NO}_{3}-\mathrm{N}$ and atrazine through soil columns as affected by lime application. Transactions of the ASAE, St. Joseph, v.39, n.3, p.937-945, 1996. 
Costa, S. N. Desenvolvimento de um modelo computacional para simular o transporte de água e solutos no solo sob condições de escoamento não permanente na vertical. Viçosa: UFV. 1998. 153p. Tese Doutorado

Dou, Z.; Fox, R. H. Using NCSWAP to simulate seasonal nitrogen dynamics in soil and corn. Plant and Soil, Derdrecht, v.177, p.235-247, 1995.

EMBRAPA - Empresa Brasileira de Pesquisa Agropecuária. Manual de métodos de análise de solo. Rio de Janeiro: Centro Nacional de Pesquisas de Solos/CNPS, 1997. 212p. Documento 1

Faria, R. T. de; Madramootoo, C. A. Simulation of soil moisture profiles for wheat in Brazil. Agricultural Water Management, Amsterdan, v.31, n.1, p.35-49. 1996.

Johnson, A. D.; Cabrera, M. L.; Mccracken, D. V.; Radcliffe, D. E. Leach simulations of nitrogen dynamics and water drainage in the ultisol. Agronomy Journal, Madison, v.91, n.4, p.597606, 1999.

Lemley, A. T.; Wagenet, R. J.; Zhong, W. Z. Sorption and degradation of aldicarb and its oxidation products in a soilwater flow system as a function of $\mathrm{pH}$ and temperature. Journal of Environmental Quality, Madison, v.17, n.3, p.508-414, 1988.

Liwang, M.; Scott, H. D.; Shaffer, M. J.; Ahuja, L. R. RZWQM simulations of water and nitrate movement in a manured tall fescue field. Soil Science, Baltimore, v.163, n.4, p.259-270, 1998.

Oliveira, L. F. C. de; Martinez, M. A.; Pruski, F. F.; Ruiz, H. A.; Lima, L. A. Transporte de solutos no solo e no escoamento superficial: I - desenvolvimento do modelo e simulação do movimento de água e escoamento superficial. Revista Brasileira de Engenharia Agrícola e Ambiental, Campina Grande, v.4, n.1, p.63-69, 2000.
Oliveira, P. C.; Lima, J. L. Simulação numérica de movimento de água em solo não saturado. Revista Brasileira de Engenharia Agrícola e Ambiental, Campina Grande, v.7, n.2, p.251257, 2003.

Sagardoy, J. A. Una visión global de la contaminación del água por la agricultura. In: Prevención de la contaminación del agua por la agricultura y actividades afines. Santiago: FAO, 1993. p.19-26.

Sassner, M.; Jensen, K. H.; Destouni, G. Chloride migration in heterogeneous soil. 1: experimental methodology and results. Water Resources Research, Washington, v.30, n.3, p.735-745, 1994.

Tillotson, W. R.; Wagenet, R. J. Simulation of fertilizer nitrogen under cropped situations. Soil Science, Baltimore, v.133, n.3, p.133-143, 1982.

Vanclooster, M.; Viaene, P.; Diels, J.; Christiaens, K. Wave, a mathematical model for simulating water and agrochemicals in the soil and vadose environment. Leuven: Institute for Land and Water Management, 1994. 145p.

Vogel, T.; Huang, K.; Zhang, R.; van Genuchten, M. Th. The HYDRUS code for simulating one-dimensional water flow, solute transporte, and heat movement in variably-saturated media. Riverside: United States Salinity Laboratory, 1996. 131p. Research Report, 140

Wagenet, R. J.; Hutson, J. L. Computer simulation models as an aid in estimating the probability of pesticide leaching, In: International Conference on Computer in Agriculture, 5, Orlando, Proceeding... Orlando: ASAE, 1994. p. 786-794.

Wang, F.; Bear, J.; Shaviv, A. A n-dynamics model for predicting n-behavior subject to environmentally friendly fertilization practices: II - numerical model and model validation. Transport in Porous Media, v.33, p.309-324, 1998. 